\title{
Aplicación de las técnicas \\ cromatográficas al análisis químico de los Bienes Culturales
}

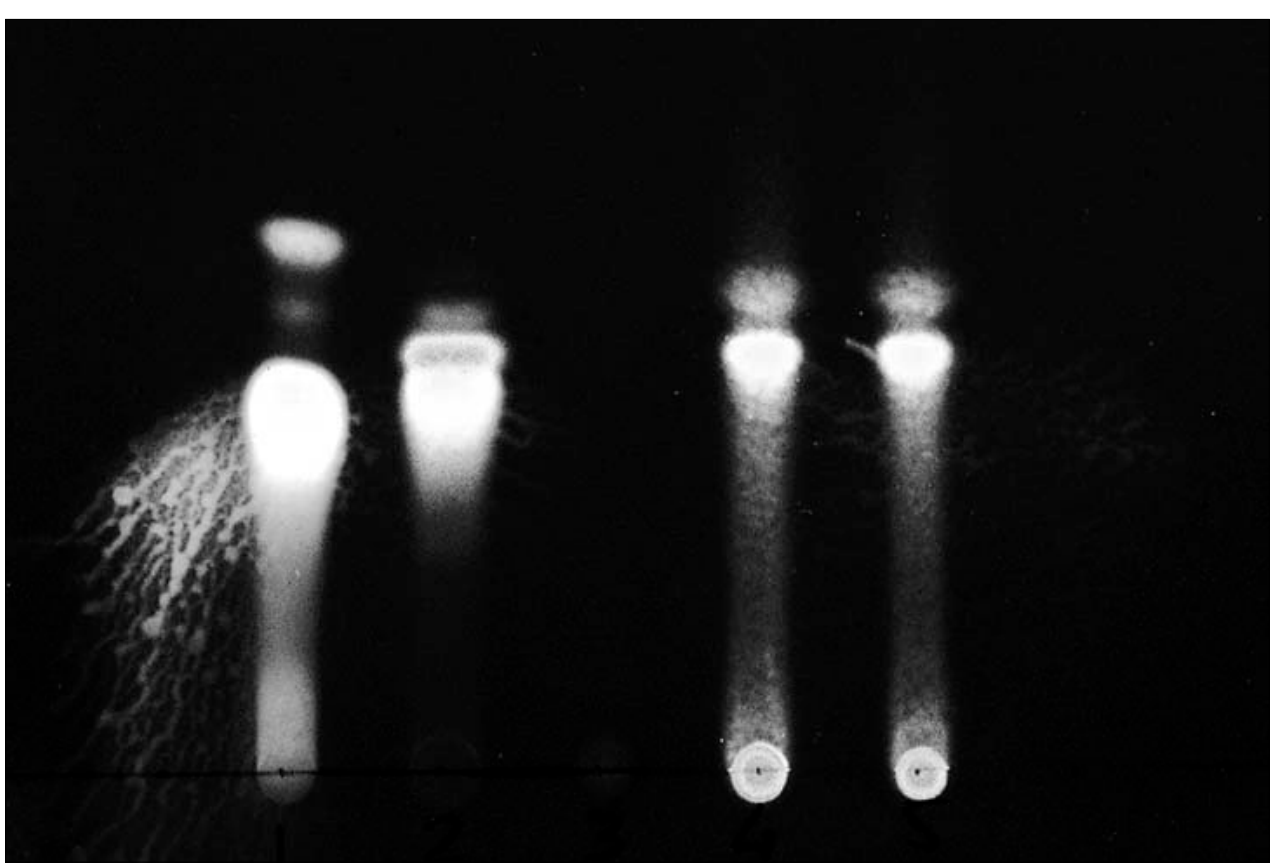

Francisco Gutiérrez Montero Asesor Técnico-Laboratorio Departamento de Análisis Centro de Intervención del IAPH a cromatografía es una técnica analítica descrita por primera vez a principios de siglo por el botánico ruso Tswett, que la aplicó a la separación de pigmentos de plantas. Le dio el nombre de cromatografía haciendo referencia a las bandas coloreadas de pigmentos que se separaban por su adsorción selectiva sobre sulfato cálcico. El uso de la técnica se fue extendiendo, a la vez que se desarrollaban distintas versiones: cromatografía de reparto, de papel, de capa fina, etc. Un momento importante en la historia de la cromatografía lo constituyó el desarrollo de la cromatografía gas-líquido (Martin y James, 1952). Esta técnica encontró rápidamente aplicaciones de gran importancia, lo que llevó al desarrollo de la teoría de la separación cromatográfica (Van Deemter, 1956; Giddings, 1965, etc.) así como al desarrollo de una instrumentación que permitía un mayor control de las condiciones de trabajo.

Actualmente no existe campo de la química, biología, medicina, etc. en el que no se utilice la cromatografía 
$\longleftarrow$
$\square$

Fig. 1.

Algunos de los principales colorantes naturales<smiles>O=C1c2ccccc2C(=O)c2c1ccc(O)c2O</smiles>

ALIZARINA<smiles>O=C(O)c1c(O)c(O)c2c(c1O)C(=O)c1ccccc1C2=O</smiles>

PSEUDOPURPURINA<smiles>O=C1CC(c2ccc(O)c(O)c2)Oc2cc(O)cc(O)c21</smiles>

LUTEOLINA en alguna de sus formas, tanto en su vertiente preparativa como en la analítica.

Las técnicas cromatográficas también tienen aplicación en el análisis químico de los bienes culturales, concretamente en el análisis de compuestos orgánicos. Estos compuestos orgánicos aparecen como aglutinantes, adhesivos, consolidantes y barnices de capas pictóricas y como colorantes de fibras textiles.

Las distintas técnicas cromatográficas se basan en la separación de los componentes de una mezcla por reparto entre dos fases: una fase estacionaria y una fase móvil. Dependiendo del tipo de fases utilizadas aparecen los distintos tipos de cromatografía. Se considera a<smiles>O=C1c2ccccc2C(=O)c2c(O)c(O)cc(O)c21</smiles>

PURPURINA<smiles>Cc1c(C(=O)O)c(O)cc2c1C(=O)c1c(O)cc(O)c(O)c1C2=O</smiles>

ACIDO KERMÉSICO<smiles>O=C1C(=C2Nc3ccccc3N2)C(=O)c2ccccc21</smiles>

INDIGO<smiles>Cc1c(C(=O)O)c(O)cc2c1C(=O)c1c(O)c(C3OC(CO)C4OC3C(O)C4O)c(O)c(O)c1C2=O</smiles>

ACIDO CARMINICO continuación la aplicación de la cromatografía en capa fina a la identificación de colorantes naturales en fibras textiles y la cromatografía en fase gaseosa a la identificación de aglutinantes y barnices en capas pictóricas.

\section{Cromatografía en capa fina}

La cromatografía en capa fina es aquella que utiliza como fase estacionaria una capa fina (de 0.25 a $2 \mathrm{~mm}$.) de sustancia adsorbente (gel de silice, alúmina o celulosa) soportada sobre alguna superficie plana (vidrio o aluminio). La separación se consigue por el movimiento capilar ascendente de la fase móvil.

El análisis por cromatografía en capa fina se realiza siguiendo la técnica de análisis por desarrollo, que consiste en aplicar las muestras a separar en forma de manchas circulares sobre uno de los extremos de la cromatoplaca, con ayuda de un microcapilar. Junto a las muestras a analizar se aplican los patrones correspondientes. A continuación se introduce la cromatoplaca en una cubeta cerrada que contiene la fase móvil (disolvente). La separación se produce por el ascenso capilar de la fase móvil a través de la fase estacionaria. A medida que asciende el disolvente, la muestra se ve arrastrada por la fase móvil y adsorbida por la fase estacionaria. Dependiendo de la afinidad química, unos componentes serán más desplazados (mayor afinidad con la fase móvil) que otros (menor afinidad), consiguiendose la separación.

La visualización de los componentes separados se realiza por pulverización de un revelador y posterior observación de la fluorescencia bajo luz ultravioleta de $254 \mathrm{~nm}$.

Tras el desarrollo, la identificación de cada componente de la mezcla se realiza midiendo el desplazamiento relativo de cada mancha en la placa respecto al frente del disolvente, definiéndose para cada banda su valor Rf, como:

$\mathrm{Rf}$ = desplazamiento del compuesto / desplazamiento del frente del disolvente

Los valores Rf, así como la fluorescencia bajo luz ultravioleta, se comparan con la de los patrones correspondientes.

Aplicación de la cromatografía en capa fina a la identificación de colorantes naturales

Las aplicaciones de la cromatografía en capa fina para el análisis químico de bienes culturales son numerosas. Se considera la identificación de colorantes naturales en fibras textiles.

\section{Colorantes naturales}

Los colorantes naturales provienen tanto de plantas como de insectos, aunque estos últimos son única- 
mente la fuente de colorantes rojos. Están restringidos a rojos, amarillos, azules y marrones. El resto de colores se obtiene de la mezcla entre ellos o de la utilización de diferentes mordientes.

\section{Colorantes rojos}

Los principales colorantes rojos de origen natural son:

Granza, obtenida de las raíces de la planta Rubia tinctorum y otras especies. Su color rojo es debido a la presencia de diferentes componentes, todos ellos derivados antraquinónicos, cuyas proporciones pueden variar dependiendo de la procedencia de la planta y del proceso de extracción del colorante. Los tres componentes principales son alizarina, purpurina y pseudopurpurina.

Kermes, obtenido de las hembras del insecto Kermes illicis L. El principal componente es ácido kermésico.

Cochinilla americana, obtenido del insecto Dactylopius coccus. El principal componente es ácido carmínico.

Otros colorantes rojos de origen vegetal son:

Palo Brasil, obtenido de distintas especies de Caesalpinia. La principal sustancia colorante es la brasileina.

Orchilla, obtenido de distintas especies de líquenes (Roccella). El componente principal es la orceína.

\section{Colorantes amarillos}

Son fundamentalmente compuestos flavonoides, ampliamente distribuidos en el reino vegetal. Los principales colorantes son:

Gualda, obtenida de la planta Reseda luteola. El componente principal es la luteolina.

Zumaques o fustetes obtenidos de las plantas Chlorophora tinctoria y Rhus cotinus. Contienen morina y fisetina.

Quercitrón, obtenido del Quercus tinctoria. El componente principal es quercitina.

Azafrán, obtenido de la planta C. sativus. El componente principal es crocetina.

Bayas persas, obtenidas de distintas especies de Rhamnus. Contienen ramnetina, quercitina y hemodina

\section{Colorantes azules}

El principal colorante azul es el índigo obtenido de distintas especies de Indigofera. El componente principal es la indigotina.

\section{Identificación de colorantes naturales}

El análisis por cromatografía en capa fina de colorantes naturales comprende las siguientes etapas:
I. Extracción del colorante de la fibra.

$\varangle$

La extracción del colorante de la fibra se realiza mediante hidrólisis ácida en medio alcohólico (ácido clorhídrico / metanol). En estas condiciones se rompen los enlaces fibra-mordiente-colorante y se consigue la disolución del colorante.

2. Purificación del colorante extraído.

3. Desarrollo cromatográfico

Las muestras y los patrones correspondientes se aplican sobre uno de los extremos de la cromatoplaca (fase estacionaria: gel de sílice 60 con indicador de fluorescencia a $254 \mathrm{~nm}$ ) con ayuda de un microcapilar de I I. A continuación se introduce en una cubeta cerrada y en atmósfera saturada de fase móvil (tolueno / formiato de etilo / ácido fórmico en la proporción 5: 4: I $\mathrm{v} / \mathrm{v}$ ). El desarrollo cromatográfico concluye cuando el frente de disolvente alcanza el extremo superior de la cromatoplaca.

Se evapora el disolvente y se rocía con revelador (2-aminoetildifenilborato en metanol). A continuación se observa la fluorescencia bajo luz ultravioleta de $254 \mathrm{~nm}$.

La comparación entre los desarrollos cromatográficos de la muestra y los patrones permite la identificación del colorante utilizado.

Se ilustra con dos separaciones:

A) Identificación de colorantes rojos:

En la Fig.2 se observa la aplicación de patrones y muestras sobre la línea base de la cromatoplaca. Constituye el momento de inicial de la separación cromatográfica. De izquierda a derecha:

I. Patrón de alizarina

2. Patrón de purpurina

3. Patrón de cochinilla americana

4. Patrón de kermes

5. Muestra de hilo rojo del Pendón de las Navas de Tolosa

6. Muestra de hilo rojo del Pendón de San Fernando

En la Fig. 3 se aprecia el momento final del desarrollo cromatográfico con observación bajo luz visible.
Fig. 2.

Cromatografía en capa fina de colorantes rojos. Aplicación de patrones y muestras

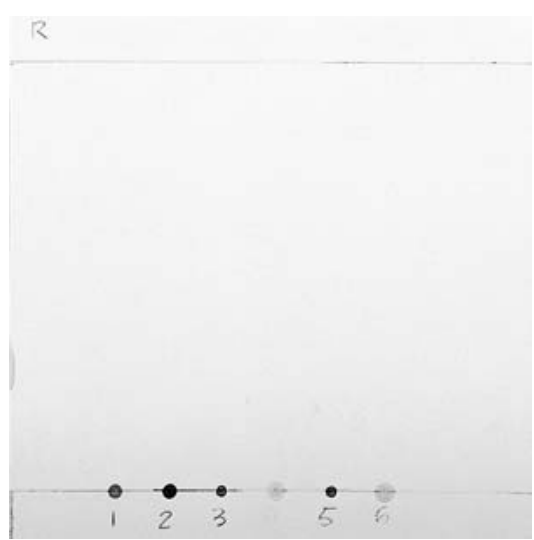

Fig. 3.

Final del desarrollo cromatográfico. Observación con luz visible R

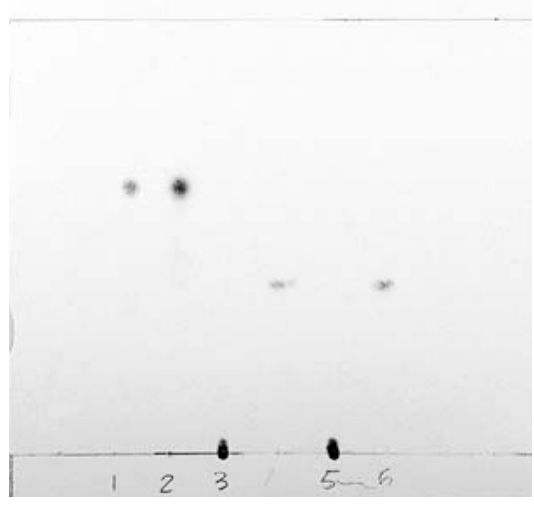

Fig. 4.

Final del desarrollo cromatográfico.

Observación con luz ultravioleta

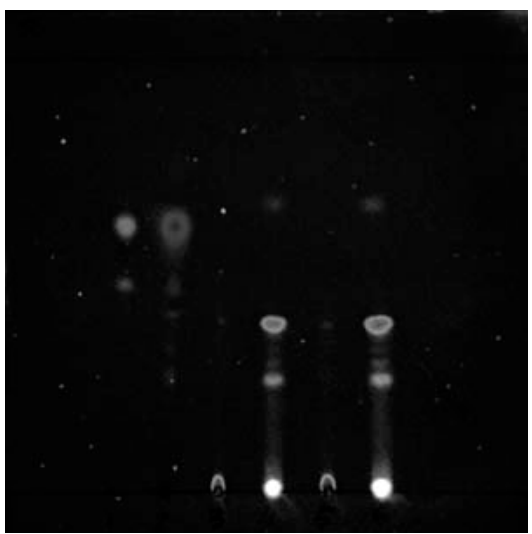


$\longleftarrow$
$\square$
-

Fig. 5.

Cromatografía en capa fina de colorantes amarillos. Aplicación de patrones y muestra

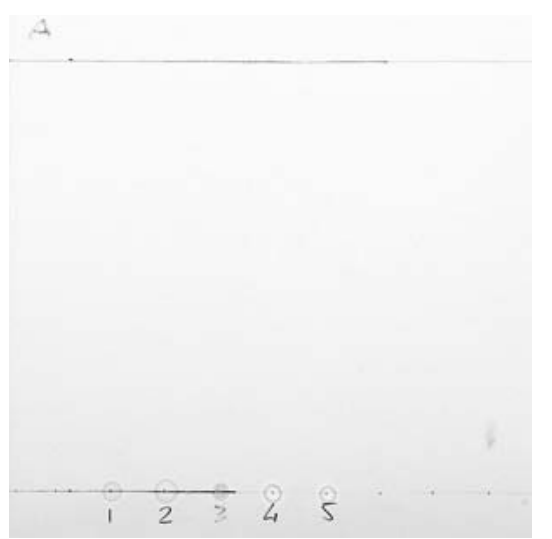

Fig. 6.

Final del desarrollo cromatográfico.

Observación con luz visible
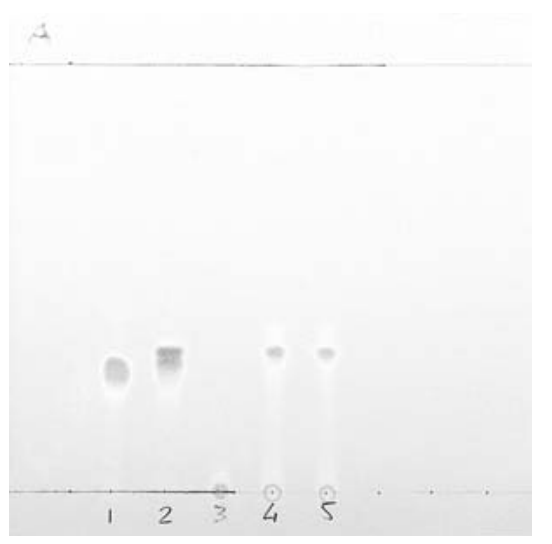

Fig. 7.

Final del desarrollo cromatográfico.

Observación con luz ultravioleta

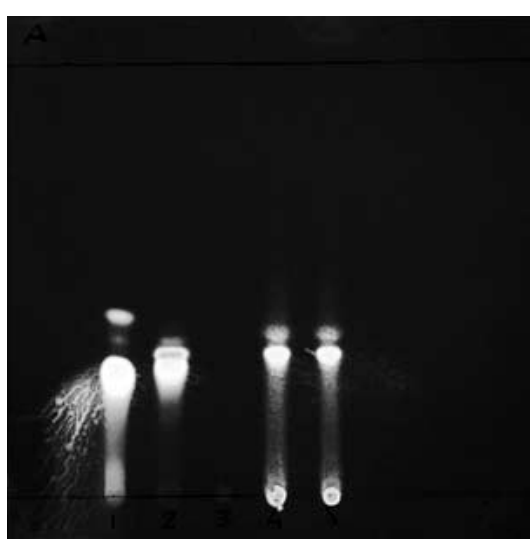

B) Identificación de colorantes amarillos

En la Fig. 5 se observa la aplicación de patrones y muestra sobre la línea base de la cromatoplaca. Constituye el momento inicial de la separación cromatográfica. De izquierda a derecha:

I. Patrón de morina

2. Patrón de fisetina

3. Patrón de azafrán

4. Patrón de gualda

5. Muestra de hilo amarillo del Pendón de San Fernando

En la Fig. 6 se aprecia el momento final del desarrollo cromatográfico con observación bajo luz visible.

En la Fig. 7 se aprecia el momento final del desarrollo cromatográfico con observación bajo luz ultravioleta de $254 \mathrm{~nm}$.

Se observa que el colorante amarillo del Pendón de San Fernando es gualda.

\section{Cromatografía en fase gaseosa}

La cromatografía en fase gaseosa es una técnica de separación en la que la fase móvil es un gas, denominado gas portador. Las muestras a separar son gases o líquidos fácilmente volatilizables. La fase estacionaria suele ser un líquido de baja volatilidad, que recubre el interior de la columna (columna capilar) o la superficie de pequeñas partículas que están rellenando la columna (columna empaquetada).

Al igual que en todos los métodos cromatográficos, la separación en cromatografía de gases se debe a la migración diferencial de los componentes de una mezcla a través de la columna. Hay un fenómeno de reparto entre dos fases, la fase gaseosa móvil (gas portador), y la fase líquida estacionaria (en la columna). En una mezcla de sustancias, que tengan polaridades similares, pero distinta volatilidad, los componentes más volátiles están más tiempo en la fase móvil, y por lo tanto, se desplazan por la columna más rápidamente que los menos volátiles, que están más tiempo en la fase estacionaria líquida.

El funcionamiento de un cromatógrafo de gases es el siguiente:

Un líquido volátil se inyecta, a través de un septum de goma, en el inyector, que se calienta para vaporizar la muestra. El gas portador (suele ser helio o nitrógeno) arrastra la muestra vaporizada hacia la columna, que es donde tiene lugar la separación. La columna está dentro de un horno, cuya temperatura puede controlarse para favorecer la separación. Después, los componentes separados salen de la columna y van al detector. El sistema de detección debe ser capaz de señalar la elución de un componente de la muestra y ofrecer al mismo tiempo una señal proporcional a la cantidad de sustancia que pasa por el detector. Los detectores utilizados en cromatografía de gases son de tipo diferencial, no ofrecen señal cuando pasa por ellos solamente gas portador y miden alguna propiedad que varíe cuando el gas portador se encuentra mezclado con la sustancia eluida de la columna.

El detector más utilizado para la identificación de compuestos orgánicos es el de ionización de llama (FID). En este detector se genera una llama con hidrógeno y aire que produce la combustión de los componentes separados que van saliendo de la columna. El sistema de detección mide los cambios en la conductividad eléctrica de la llama de forma que cuando sólo sale gas portador la señal es mínima y aumenta al salir un componente de la muestra. Este aumento en la señal del detector es proporcional a la cantidad de muestra separada.

La señal del detector, debidamente amplificada y tratada, se representa gráficamente frente al tiempo de retención (tiempo transcurrido desde la inyección de la muestra hasta la salida por el detector) obteniéndose el cromatograma. El cromatograma consiste en una serie de picos con forma gaussiana a distintos tiempos de retención.

Actualmente los cromatógrafos de gases van acoplados a un ordenador que realiza el control de todas las variables del sistema y la adquisición y tratamiento de los datos.

\section{APLICACIONES DE LA CROMATOGRAFÍA EN FASE GASEOSA. IDENTIFICACIÓN DE AGLUTINANTES Y BARNICES EN CAPAS PICTÓRICAS}

\section{Identificación de aglutinantes}

Los compuestos orgánicos que pueden aparecer en las capas pictóricas y de preparación deben su uso a sus propiedades filmógenas (son sustancias que se aplican en estado líquido y al secar son capaces de for- 
mar capas o películas), utilizándose para la preparación de aglutinantes, adhesivos, consolidantes y barnices. Corresponden a los siguientes grupos de sustancias: proteínas, polisacáridos, ceras, aceites secantes, resinas naturales, materiales bituminosos y sintéticos.

Vamos a considerar la aplicación de la cromatografía en fase gaseosa a la identificación de los dos grupos principales de aglutinantes: proteínas y aceites secantes.

\section{Identificación de aglutinantes proteicos}

Las proteínas están constituidas por polímeros naturales formados por la unión de aminoácidos mediante enlace peptídico. En su composición entran los L-aminoácidos siguientes: histidina, triptófano, lisina, hidroxiprolina, arginina, ácido aspártico, ácido glutámico, glicina, alanina, valina, isoleucina, leucina, serina, treonina, prolina, fenilalanina, tirosina, cistina, metionina.

La diferenciación entre los tres tipos fundamentales de sustancias proteicas utilizadas en restauración (colas animales, huevo y caseína) estriba en las distintas proporciones de aminoácidos que entran en su composición, por lo que la identificación del tipo de proteína implica el análisis de los aminoácidos que las componen.

El procedimiento analítico consta de las siguientes etapas:

I. Hidrólisis de la proteína para liberar los aminoácidos al medio

Este proceso suele realizarse con ácido clorhídrico $6 \mathrm{~N}$ durante 24 h. a $110^{\circ} \mathrm{C}$.

\section{Purificación del hidrolizado}

Además de aminoácidos, el hidrolizado contiene impurezas (sales, carbohidratos, etc.) que deben ser eliminadas. La eliminación de impurezas se consigue por filtración del hidrolizado a través de una resina de intercambio iónico.

\section{Derivación}

Un requerimiento fundamental para poder analizar una muestra por cromatografía en fase gaseosa es que la muestra sea volátil. Caso de no serlo, como ocurre con los hidrolizados proteicos, hay que recurrir a la obtención de derivados volátiles.

Existen varios procedimientos para la obtención de derivados volátiles de aminoácidos. El procedimiento analítico utilizado en el Laboratorio de Química del I.A.P.H. implica la obtención de trimetilsililderivados de aminoácidos, mediante la utilización del reactivo derivante bis-trimetilsililacetamida.

4. Inyección de la muestra en el cromatógrafo de gases

Una vez obtenido el derivado volátil, se introduce la muestra en el sistema de inyección del cromatógrafo con ayuda de una microjeringa.

\section{Separación de los aminoácidos}

La muestra inyectada contiene los derivados volátiles de los aminoácidos que componen la proteína objeto de identificación. La separación de estos aminoácidos se consigue en las siguientes condiciones:

Fase móvil: $\quad$ Nitrógeno, presión en cabeza de columna 25 psi.

Fase estacionaria: Polidimetilsiloxano (columna HP-1, $30 \mathrm{~m}, 0.25 \mu \mathrm{m}, 0.25 \mathrm{~mm}$ d.i.)

Programa de temp: De $90^{\circ} \mathrm{C}$ a $220^{\circ} \mathrm{C}$, gradiente $2^{\circ} \mathrm{C} / \mathrm{min}$.

Inyección: $\quad$ Splitless a $250^{\circ} \mathrm{C}$.

Detector: $\quad \quad \quad F I D$ a $250^{\circ} \mathrm{C}$

Fig. 8.

Separación e identificación de aminoácidos por cromatografía en fase gaseosa

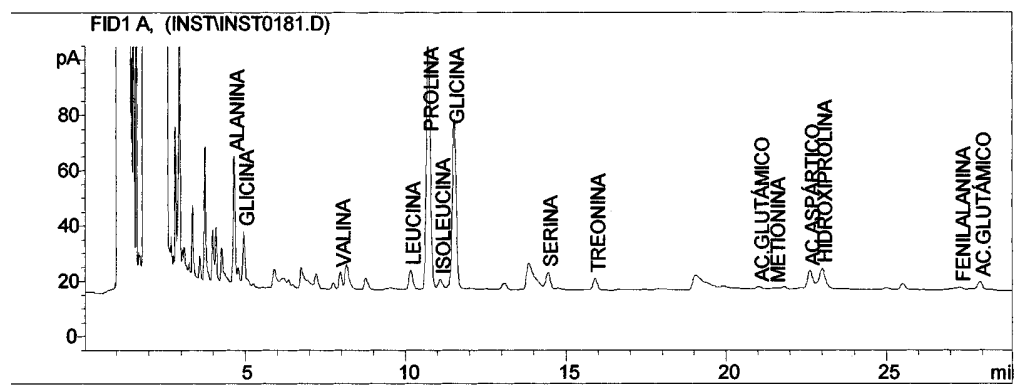

Fig. 9.

Separación e identificación de ácidos grasos por cromatografía en fase gaseosa

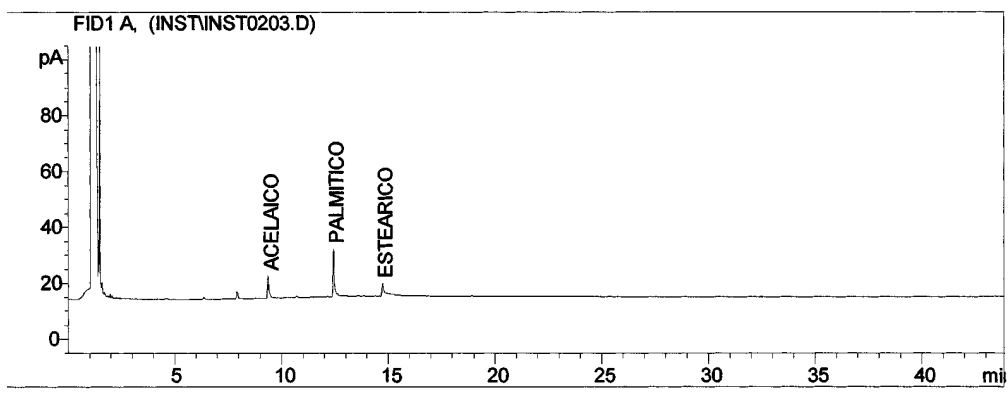

Las colas animales se caracterizan por la presencia del aminoácido L-hidroxiprolina, no presente en el huevo ni en la caseína, asociada a altos niveles de L-prolina y L-glicina (20\%).

El huevo se caracteriza por su alto contenido en L-serina (10\%) y la presencia de grasas en la yema. Mientras que la caseína presenta niveles de ácido L-glutámico superiores al $20 \%$.

En la Fig. 8 se observa el cromatograma de una capa de preparación de la obra $\mathrm{D}^{\mathrm{a}}$ Leonor y $\mathrm{D}^{\mathrm{a}}$ Mencia de Cabrera e hijas de Pedro de Campaña. La distribución 
땀 de aminoácidos es acorde con una cola animal (presencia de L-hidroxiprolina asociada a altos niveles de L-prolina y L-glicina).

\section{Identificación de aglutinantes oleosos}

Desde el punto de vista químico los aceites están constituidos por triglicéridos de ácidos grasos. Un triglicérido es un éster formado por la reacción de esterificación entre un alcohol (glicerina) y ácidos grasos. Los ácidos grasos que entran en la composición del triglicérido son:

Acidos grasos saturados: ácidos palmítico y esteárico.

Fig. 10.

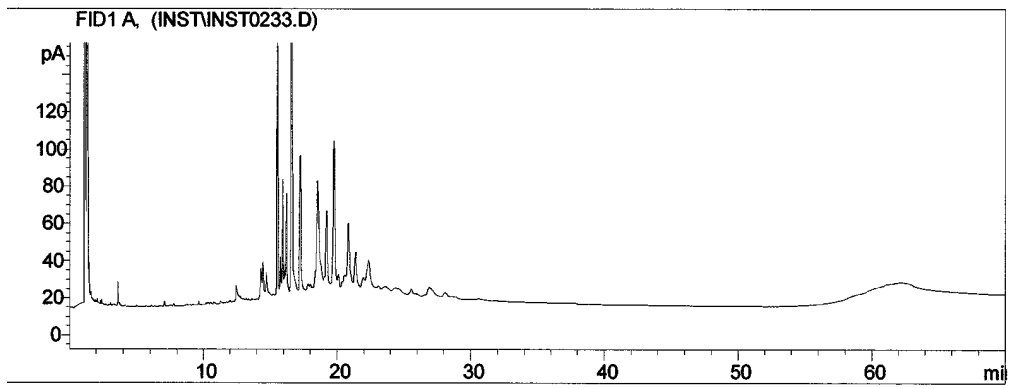

Fig. 11.

Cromatografía de almáciga

\section{FID1A (INSTUNSTO232.D)}

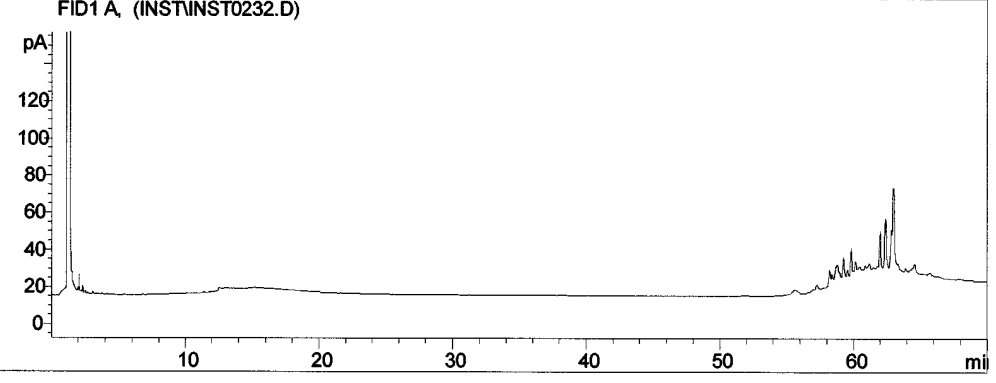

Fig. 12.

\section{Cromatograma de dammar}

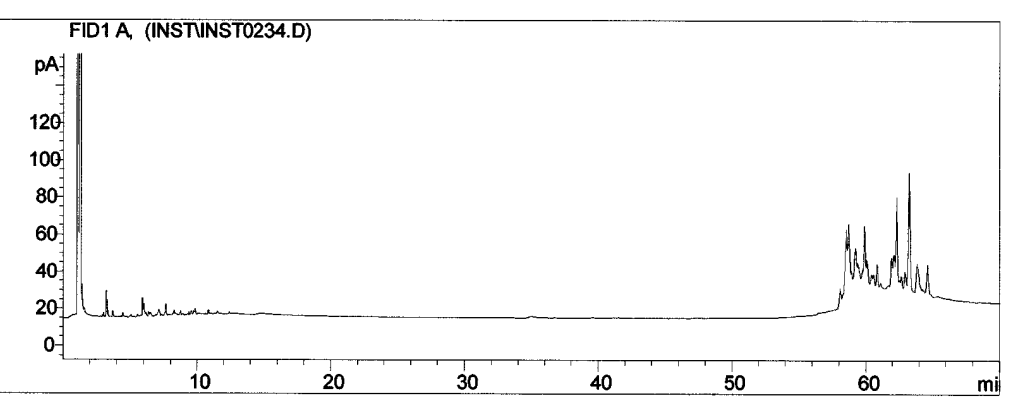

Acidos grasos insaturados: ácidos oleico, linoleico y linolénico.

Los principales aceites secantes utilizados en restauración son aceite de lino, de nueces y de adormidera. La diferenciación entre estos tres tipos de aceites implica el análisis de los ácidos grasos que los componen.

Los ácidos grasos insaturados (oleico, linoleico y linolénico) son los responsables de las propiedades secantes de los aceites ya que a través de transformaciones químicas, principalmente de oxidación, conducen a la formación del polímero linoxina. La destrucción de este polímero conduce la formación de ácido acelaico, ácido dicarboxílico no presente en la composición original del aceite.

El procedimiento analítico para la identificación de aceites implica las siguientes etapas:

I. Hidrólisis del triglicérido para liberar los ácidos grasos.

2. Preparación de los derivados volátiles de los ácidos grasos para poder ser inyectados en el cromatógrafo.

Al igual que ocurría en el caso de los aminoácidos, los ácidos grasos no son volátiles por lo que hay que recurrir a la preparación de derivados volátiles. Existen varios procedimientos para la obtención de derivados volátiles de ácidos grasos. El procedimiento utilizado en el Laboratorio de Química del I.A.P.H. supone la obtención de trimetilsililderivados de ácidos grasos mediante la reacción con el reactivo derivante bis-trimetilsililacetamida.

3. Inyección de la muestra

4. Separación de ácidos grasos

La separación de ácidos grasos se consigue en las siguientes condiciones:

Fase móvil: $\quad$ Nitrógeno, presión en cabeza de columna 25 psi.

Fase estacionaria: Polidimetilsiloxano (columna HP-1, $30 \mathrm{~m}, 0.25 \mu \mathrm{m}, 0.25 \mathrm{~mm}$ d.i.)

Programa de temp: De $150^{\circ} \mathrm{C}$ a $230^{\circ} \mathrm{C}$, gradiente $10^{\circ} \mathrm{C} / \mathrm{min}$.

Inyección: $\quad$ Splitless a $230^{\circ} \mathrm{C}$.

Detector: $\quad \quad \quad F I D$ a $250^{\circ} \mathrm{C}$.

En el cromatograma de aceites se observan fundamentalmente los ácidos palmítico, esteárico y acelaico. La presencia del ácido acelaico es consecuencia del proceso de secado del aceite. Las diferencias entre los tres tipos de aceites utilizados en restauración están en las distintas proporciones de ácidos palmítico y esteárico.

Palmítico / Esteárico

\begin{tabular}{ll}
\hline Aceite de lino & $0.7-1.4$ \\
Aceite de nueces & $2.5-3.5$ \\
Aceite de adormidera & $4.0-5.0$
\end{tabular}


En la Fig. 9 se observa el cromatograma de una policromía roja del Retablo de la Iglesia de la Encarnación de Albolote (Granada). Se detectan los ácidos acelaico, palmítico y esteárico. La relación ácido palmítico / ácido esteárico (1.2) se corresponde con un aceite de lino.

\section{IDENTIFICACIÓN DE BARNICES}

La cromatografía en fase gaseosa también tiene aplicación en la identificación de las resinas naturales empleadas en la formulación de barnices. Estas resinas están constituidas por polímeros derivados del isopreno. En su composición aparecen ácidos e hidrocarburos terpénicos. Pueden clasificarse en dos grandes grupos:
Resinas diterpénicas, también denominadas resinas duras: colofonia, sandaraca, ámbar.

Resinas triterpénicas, también denominadas resinas blandas: almáciga, dammar.

El procedimiento analítico empleado para la identificación de estas resinas es similar al descrito para la identificación de aceites secantes.

En las Fig. 10, 11 y 12 se observan los cromatogramas de colofonia, almáciga y dammar, respectivamente.

\section{Bibliografía}

GÓMEZ GONZÁLEZ Mª L. Examen científico aplicado a la conservación de obras de arte. Ministerio de Cultura 1994.

PARRA, E. Análisis de materiales orgánicos. Técnicas y aplicaciones Curso de técnicas de diagnóstico aplicadas a la conservación de los bienes muebles. Granada 1996

C. J. SERNA. Espectroscopía IR, visible y UV. Cromatografía. Introducción a la ciencia de materiales: técnicas de preparación y caracterización. CSIC 1993

KLEBE J. F., FINKBEINER H. and WHITE D. M. Silylations with bis(trimethylsilyl)acetamide, a highly reactive silyl donor. Journal of the American Chemical Society $n^{\circ}$ 20, 1966.

MILLS J. S. and WHITE R. The organic chemistry of museum objects. Ed. Butterworths, 1987.

MILLS J. S., WHITE R. The gas-chromatographic examination of paint media. Some examples of medium identification in paintings by fatty acid analysis. Conservation and restoration of pictorial art. Ed. N. Brommelle and P. Smith. 1976.

WHITE R. The characterization of proteinaceous binders in art objects. National Gallery Technical Bulletin n 8, 1984.
PANCELLA R., BART R., FURLAN V. Application de la chromatographie en phase gazeuse à l'identification des matières organiques dans les couches picturales. Methods for the preservation of cultural properties. Ed. Schweizer and Villiger. 1989

DE PABLOS F. Cromatografía en fase gaseosa. Prácticas de Laboratorio. Departamento de Química Analítica. Universidad de Sevilla.

MASSCHELEIN-KLEINER L. Contribution to the study of aged proteinaceous media. Conservation and restoration of pictorial art. Ed. N. Brommelle and P. Smith. 1976.

MASSCHELEIN-KLEINER L, HEYLEN J. et TRICOT-MARCKX. Contribution à I'analyse des liants, adhésifs et vernis anciens. Studies in Conservation $n^{\circ} 13,1968$.

MASSCHELEIN-KLEINER L, MAES L. and ZRAMENSKI FESTRATES N. Etude technique de la tapisserie tournaisienne au XVe siecle. Les colorants. Bulletin de I'IRPA XI, 1969.

HOFENK-DE GRAAFF J. $\mathrm{H}$. and ROELOFS W. G.Th. On the occurrence of red dyestuffs in textile materials from the period 1450 1600. ICOM Committee for Conservation 3rd Triennial Meeting. Madrid 1972. 